\title{
Advancing TEM Based Biomedical Nanoparticle Characterization: GMP validated TEM Workflow In a BSL2 Environment with CNN as Automated Analytical Tool
}

Mathieu Colomb-Delsuc ${ }^{1}$, Johan Härmark ${ }^{2}$, Max Philström ${ }^{2}$, Rickard Nordström ${ }^{2}$, Gustaf Kylberg ${ }^{2}$ and Ida-Maria Sintorn ${ }^{3}$

${ }^{1}$ Vironova AB, Stockholm, Stockholms Lan, Sweden, ${ }^{2}$ Vironova AB, United States, ${ }^{3}$ University of Uppsala, United States

Transmission Electron Microscopy (TEM) is routinely used for the characterization of nano-sized particles in life sciences. Among the variety of specimen analyzed, some involve risks and require strict safety procedures when being handled. Working with infectious and pathogenic agents such as some viruses, vaccines or gene therapy vectors for example, implies to operate in contained facilities with a well-controlled environment.

Some of the requirements for working in Biosafety Level 2 (BSL2) conditions, as described by the European Commission guidelines [1], are quite challenging to fulfill within the standard TEM workflow. Consequently, the daily routines need to be adapted in order to combine the challenges of TEM with safety requirements, such as performing precision work with bulky gowning, preparing the specimen and transferring liquid nitrogen inside a facility with a high air-flow, or decontaminating the equipment used for the specimen preparation with corrosive agents [2]. To answer the BSL2 demands, established standard protocols need to be implemented and validated, as well as access control to trained personnel exclusively. This ensures reliability and traceability of the results all along the process, from the specimen reception until the report making and archiving of data.

Construction and establishment of such a BSL2 TEM facility (Figure 1) including a Good Manufacturing Practice (GMP) validated TEM workflow had not been described to date, yet it proves to be tremendously relevant, especially for e.g., the vaccine and gene therapy domains. In these fields new requirements from the United States Pharmacopeial Convention (USP) and the U.S. Food and Drug Administration (FDA) place stress on investigating the impact of changes on the product during process and product development. New demands in product characterization involve morphological description of the particles present in the formulations, typically nanoparticles like viruses, virus-like particles and adjuvants in the range of $0.01-0.1 \mu \mathrm{m}$ [3]. TEM offers the means to answer these questions in a direct way. In addition, when entering the production phase of such products, TEM provides excellent means for production monitoring and quality control. Therefore, being able to prepare and image specimen in a controlled environment while respecting the safety guidelines also for BSL2 specimen is a clear need.

The work in the GMP environment is performed using a software developed in house, Vironova Analyzer Software (VAS). The software allows for data acquisition, storage in a controlled manner, and image analysis, while ensuring compliance with FDA 21 CFR Part 11 guidelines. Amongst the analytical tools at hand, machine learning solutions such as Convolutional Neural Networks (CNN) have been developed in the latest years and are now included in the workflows.

Their ease of use and limited human input make them a perfect method to be implemented in a regulatory controlled workflow.

The challenges and hurdles that were overcame to implement a GMP workflow in a BSL2 lab for the analysis of biological specimen with TEM will be presented and discussed, together with a description of 
the CNN workflows development, illustrated with case studies demonstrating the analytical capacities and application domains.
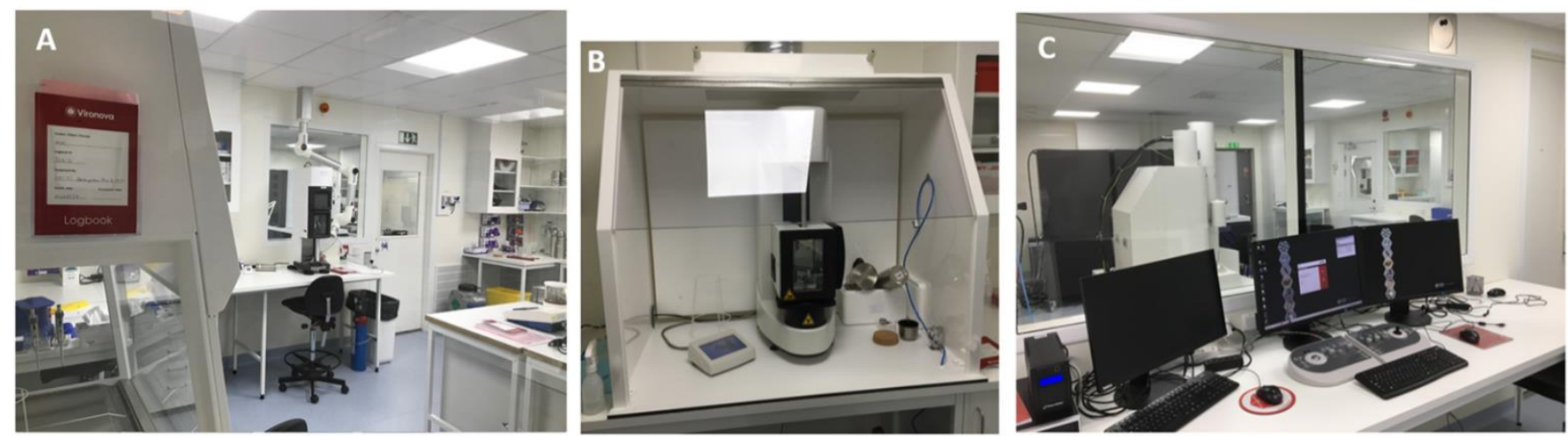

Figure 1. Figure 1. BSL2 TEM facility, with the preparation room including the biosafety cabinet (A), the plunge-freezing station (B), and the TEM control room (C).

\section{References}

[1] European law website, https://eur-lex.europa.eu/legal-content/EN/TXT/?uri=CELEX:32000L0054 (accessed 25 Feb 2021)

[2] MB Sherman et al., Journal of Structural Biology 181 (2013), p. 223

[3] U.S. Food and Drug Administration website, https://www.gmp-

compliance.org/files/guidemgr/UCM202439.pdf (accessed 25 Feb 2021) 